\title{
Predictive anatomical factors for rupture in middle cerebral artery mirror bifurcation aneurysms
}

\author{
Homajoun Maslehaty, MD, ${ }^{1}$ Crescenzo Capone, MD, ${ }^{2,3}$ Roman Frantsev, MD, ${ }^{1}$ \\ Igor Fischer, PhD, ${ }^{4}$ Ramazan Jabbarli, MD, ${ }^{1}$ Jan F. Cornelius, MD, ${ }^{2}$ Marcel A. Kamp, MD, ${ }^{2}$ \\ Paolo Cappabianca, MD, PhD, ${ }^{3}$ Ulrich Sure, MD, PhD, ${ }^{1}$ Hans-Jakob Steiger, MD, PhD, ${ }^{2}$ and \\ Athanasios K. Petridis, MD²
}

\begin{abstract}
'Department of Neurosurgery, University Hospital Essen; '2Department of Neurosurgery, University Hospital Düsseldorf; ${ }^{4}$ Department of Neurosurgery, Statistical Analysis Office, Bio-Statistics, University Hospital Düsseldorf, Germany; and 'Division of Neurosurgery, Department of Neurosciences, Reproductive and Odontostomatological Sciences, School of Medicine and Surgery, Università degli Studi di Napoli "Federico II," Naples, Italy
\end{abstract}

\begin{abstract}
OBJECTIVE The aim of this study was to define predictive factors for rupture of middle cerebral artery (MCA) mirror bifurcation aneurysms.

METHODS The authors retrospectively analyzed the data in patients with ruptured MCA bifurcation aneurysms with simultaneous presence of an unruptured MCA bifurcation mirror aneurysm treated in two neurosurgical centers. The following parameters were measured and analyzed with the statistical software R: neck, dome, and width of both MCA aneurysms-including neck/dome and width/neck ratios, shape of the aneurysms (regular vs irregular), inflow angle of both MCA aneurysms, and the diameters of the bilateral $A_{1}$ and $M_{1}$ segments and the frontal and temporal $M_{2}$ trunks, as well as the bilateral diameter of the internal carotid artery (ICA).
\end{abstract}

RESULTS The authors analyzed the data of 44 patients ( 15 male and 29 female, mean age 50.1 years). Starting from the usual significance level of 0.05 , the Sidak-corrected significance level is 0.0039 . The diameter of the measured vessels was statistically not significant, nor was the inflow angle. The size of the dome was highly significant $(p=$ $0.0000069)$. The size of the neck $(p=0.0047940)$ and the width of the aneurysms $(p=0.0056902)$ were slightly nonsignificant at the stated significance level of 0.0039 . The shape of the aneurysms was bilaterally identical in 22 cases $(50 \%)$. In cases of asymmetrical presentation of the aneurysm shape, $19(86.4 \%)$ ruptured aneurysms were irregular and $3(13.6 \%)$ had a regular shape $(p=0.001)$.

CONCLUSIONS In this study the authors show that the extraaneurysmal flow dynamics in mirror aneurysms are nonsignificant, and the aneurysmal geometry also does not seem to play a role as a predictor for rupture. The only predictors for rupture were size and shape of the aneurysms. It seems as though under the same conditions, one of the two aneurysms suffers changes in its wall and starts growing in a more or less stochastic manner. Newer imaging methods should enable practitioners to see which aneurysm has an unstable wall, to predict the rupture risk. At the moment one can only conclude that in cases of MCA mirror aneurysms the larger one, with or without shape irregularities, is the unstable aneurysm and that this is the one that needs to be treated.

https://thejns.org/doi/abs/10.3171/2017.2.JNS162705

KEY WORDS middle cerebral artery bifurcation aneurysms; predictive factors; anatomical analysis; subarachnoid hemorrhage; vascular disorders

$\mathrm{B}$ ECAUSE the occurrence of subarachnoid hemorrhage (SAH) still has a significant socioeconomic impact and affects mostly younger patients compared with ischemic stroke, the goal of the treatment of unruptured intracranial aneurysms (UIAs) is the prevention of this disease. ${ }^{16}$ However, the risks of treatment have to be considered carefully in view of the available data concerning the natural course of UIAs.

Several large prospective studies were conducted to evaluate the risk of rupture of UIAs, and they mainly esti-

ABBREVIATIONS ICA = internal carotid artery; ICH = intracerebral hemorrhage; $M C A=$ middle cerebral artery; PHASES = population, hypertension, age, size of aneurysm, earlier SAH from another aneurysm, and site of aneurysm; SAH = subarachnoid hemorrhage; UIA = unruptured intracranial aneurysm; WFNS = World Federation of Neurosurgical Societies.

SUBMITTED October 27, 2016. ACCEPTED February 15, 2017.

INCLUDE WHEN CITING Published online August 25, 2017; DOI: 10.3171/2017.2.JNS162705. 
mate an overall very low risk of rupture at approximately $1 \%$, which increases with the size of the aneurysms. Consequently, UIAs $>10 \mathrm{~mm}$ are estimated to have a significantly higher risk of rupture. ${ }^{19,24,25}$ Nevertheless, we are faced periodically with a substantial mismatch between the size of ruptured intracranial aneurysms in patients with SAH in predominantly $<7-\mathrm{mm}$ lesions and the estimated low risk of rupture of small UIAs. ${ }^{18}$ Thus, size alone does not seem to be a reliable parameter for decision making regarding treatment of single UIAs.

Other parameters like ethnicity, the presence of hypertension, the age of the patients, the size of the lesions, previous SAH, and localization of the aneurysms were pooled together in the population, hypertension, age, size of aneurysm, earlier SAH from another aneurysm, and site of aneurysm (PHASES) score for a more accurate estimation of the risk of rupture. ${ }^{9}$

To define possible anatomical predictive factors of rupture of intracranial aneurysms, we aimed to analyze the morphology and angioarchitecture of ruptured middle cerebral artery (MCA) bifurcation aneurysms and to compare them to their unruptured mirror-image counterparts within the same patient. Through this method, we formed a homogeneous group, minimized interindividual differences of various factors-as considered in the PHASES score-and enabled a comparison of different anatomical parameters.

\section{Methods}

This is a retrospective study design with the participation of the neurosurgical and neuroradiological departments of University Hospital Essen and University Hospital Düsseldorf. The study was approved by the local ethics committees of the University of Duisburg-Essen and Düsseldorf, and it was conducted in accordance with the Guidelines for Good Clinical Practice.

We retrospectively reviewed our neurovascular databases and selected all patients with SAH caused by a ruptured MCA bifurcation aneurysm and with the simultaneous presence of an unruptured contralateral MCA bifurcation aneurysm (University Hospital Essen 2004-2015, n = 25 patients; University Hospital Düsseldorf 2005-2015, $\mathrm{n}=19$ patients - total $n=44$ patients). Patients with spontaneous SAH were admitted to the respective center either primarily via the emergency room or secondarily by referring hospitals. At admission, plain CT and CT angiography scans were performed to detect the source of the hemorrhage.

In cases of SAH accompanied by space-occupying intracerebral hemorrhage (ICH), surgery was performed immediately without further imaging. In cases with SAH alone and accompanied by small ICH, digital subtraction angiography was performed within 12 hours, followed by microsurgical treatment of the ruptured aneurysm. In cases of occlusive hydrocephalus, an external ventricular drain was inserted prior to treatment of the source of bleeding for pressure control. The location of ICH was indicative of the rupture site. In addition, the ruptured lesion was identified clearly during microsurgical aneurysm clipping in all of the presented cases.

We collected the demographic data and classified the
TABLE 1. Demographic data in 44 patients with MCA mirror bifurcation aneurysms

\begin{tabular}{cc}
\hline Demographic Data & Value \\
\hline Total & 44 \\
\hline Age & Mean $50.1 \mathrm{yrs}$ \\
\hline Sex & $15(34.1 \%)$ \\
\hline Male & $29(65.9 \%)$ \\
\hline Female & \\
\hline Rupture site & $21(47.7 \%)$ \\
\hline Rt & $23(52.3 \%)$ \\
\hline Lt & $11(25 \%)$ \\
\hline WFNS grade & $5(11.4 \%)$ \\
\hline I & $15(34.1 \%)$ \\
\hline II & $9(20.5 \%)$ \\
\hline III & $4(9 \%)$ \\
\hline V & \\
\hline Fisher grade & $5(11.4 \%)$ \\
\hline 1 & $3(6.8 \%)$ \\
\hline 2 & $15(34.1 \%)$ \\
\hline 3 & $21(47.7 \%)$ \\
\hline 4 &
\end{tabular}

SAH using the World Federation of Neurosurgical Societies (WFNS) system, and we determined the Fisher score by the appearance of SAH on CT scans (Table 1).

The vascular parameters measured using the available CT angiography and digital subtraction angiography data sets were as follows: neck, dome, and width (in millimeters) of both MCA aneurysms including neck/dome, width/ dome, and width/neck ratios; shape of the aneurysms as regular (saccular) and irregular (with 1-3 additional lobes including daughter aneurysm); and inflow angle of both MCA aneurysms (Fig. 1). Furthermore, we measured the diameters of the bilateral $A_{1}$ and $M_{1}$ segments and the frontal and temporal $\mathrm{M}_{2}$ trunks, as well as the bilateral diameter of the internal carotid artery (ICA) to reflect the general vascular condition proximal and distal to the aneurysms. Different ratios of the vascular diameters were calculated and were used for statistical analysis (Table 2).

\section{Statistical Analysis}

For statistical analysis we used the statistics software $\mathrm{R}$ (version 3.3.1, 2016). We systematically tested the following variables for the difference between the ruptured and unruptured side: dome, neck, and width of the aneurysms; the diameter of the ICA, $\mathrm{A}_{1}, \mathrm{M}_{1}, \mathrm{M}_{2}$ frontal, and $\mathrm{M}_{2}$ temporal branches (all in millimeters); inflow angle (degrees); dome/neck ratio; width/neck ratio; width/dome ratio; $\mathrm{A}_{1} / \mathrm{M}_{1}$ ratio; and $\mathrm{M}_{2}$ temporal/frontal ratio (dimensionless). Starting from the usual significance level of 0.05 for a single statistical test and assuming the variable's independence, the Sidak-corrected significance level for the 13 tests is 0.0039 .

The Shapiro-Wilk test has shown that most of the 


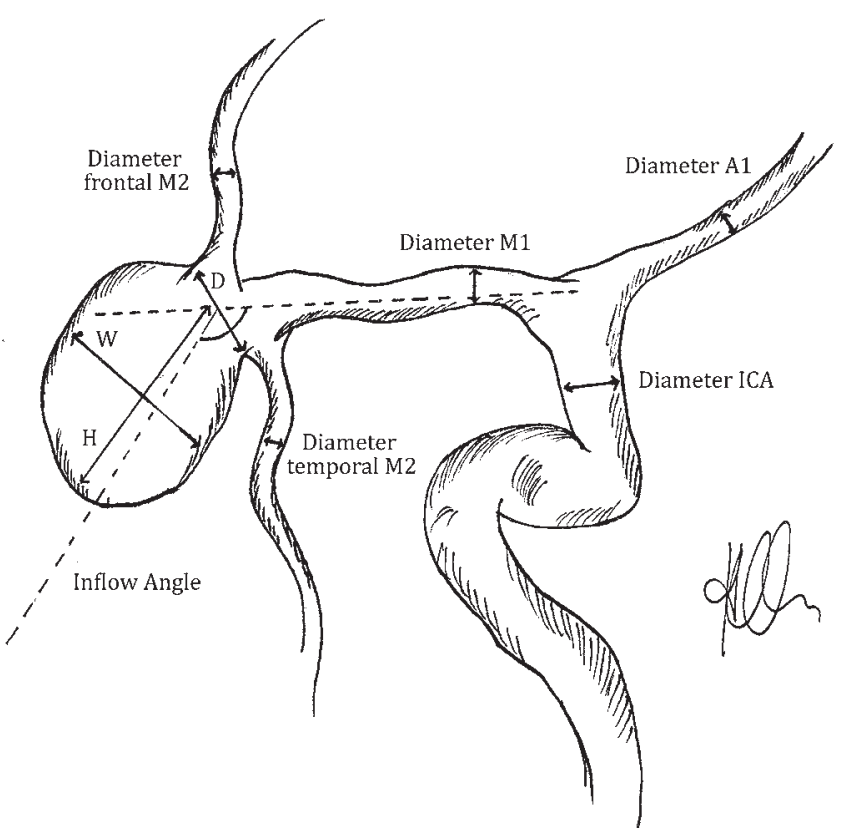

FIG. 1. Illustrative drawing of the measured vascular parameters. Copyright Homajoun Maslehaty. Published with permission.

above-mentioned variables were not normally distributed. However, their normalized differences, $2 \times(\mathrm{r}-\mathrm{u}) /(\mathrm{r}+\mathrm{u})$, where $r$ denotes a variable's value for the ruptured side and $\mathrm{u}$ for the unruptured side, were normally distributed. Therefore, we could use the t-test for the normalized variables. Such normalized differences have the advantage of being dimensionless and easily comparable, whereas original variables have different units of measure and may vary greatly in range, from $<1 \mathrm{~mm}$ for $\mathrm{M}_{2}$ to $>100^{\circ}$ for the inflow angle. For every variable we tested the null hypothesis that the normalized difference is zero, against the alternative hypothesis that it is different from zero, using the 2 -sided t-test.

\section{Results}

In total we analyzed the data in 44 patients (15 male and 29 female, mean age 50.1 years, range 32-75 years). Eleven patients $(25 \%)$ presented with SAH of WFNS Grade I, 5 patients $(11.4 \%)$ presented with WFNS Grade II, 15 patients (34.1\%) with WFNS Grade III, 9 patients (20.5\%) with WFNS Grade IV, and 4 patients (9\%) with WFNS Grade V. The results are displayed in Table 1.

The measurements of the vascular parameters and the calculated ratios are given in Table 2. Supplemental Table 1 shows the summarized data and the calculated ratios, with the Shapiro-Wilk p value in the rightmost column representing the non-normal distribution of the parameters.

Systematic testing showed that, at the above-stated significance level, only the normalized difference of the aneurysm dome is a significant factor for aneurysm rupture, whereas neck and width values are very close to significance and fail only because of Bonferroni correction. The other vascular parameters and calculated ratios were statistically nonsignificant (Table 3 ).
TABLE 2. Measurements of the vascular parameters and ratios of MCA mirror bifurcation aneurysms

\begin{tabular}{|c|c|c|c|}
\hline Variable & Min & Max & Mean \\
\hline \multicolumn{4}{|l|}{ Dome (mm) } \\
\hline Ruptured & 2.10 & 16.00 & 6.98 \\
\hline Unruptured & 1.60 & 11.10 & 4.42 \\
\hline \multicolumn{4}{|l|}{ Neck (mm) } \\
\hline Ruptured & 1.20 & 12.00 & 4.05 \\
\hline Unruptured & 1.10 & 12.00 & 2.99 \\
\hline \multicolumn{4}{|l|}{ Width (mm) } \\
\hline Ruptured & 1.60 & 15.00 & 5.29 \\
\hline Unruptured & 1.00 & 12.50 & 4.07 \\
\hline \multicolumn{4}{|l|}{$\mathrm{ICA}(\mathrm{mm})$} \\
\hline Ruptured & 2.20 & 4.90 & 3.22 \\
\hline Unruptured & 2.00 & 4.40 & 3.23 \\
\hline \multicolumn{4}{|l|}{$\mathrm{A}_{1}(\mathrm{~mm})$} \\
\hline Ruptured & 1.00 & 3.40 & 2.18 \\
\hline Unruptured & 1.30 & 3.40 & 2.08 \\
\hline \multicolumn{4}{|l|}{$\mathrm{M}_{1}(\mathrm{~mm})$} \\
\hline Ruptured & 1.70 & 4.80 & 2.40 \\
\hline Unruptured & 1.50 & 3.80 & 2.38 \\
\hline \multicolumn{4}{|l|}{$\mathrm{M}_{2}$ frontal $(\mathrm{mm})$} \\
\hline Ruptured & 0.92 & 2.40 & 1.52 \\
\hline Unruptured & 1.20 & 2.70 & 1.56 \\
\hline \multicolumn{4}{|l|}{$\mathrm{M}_{2}$ temporal $(\mathrm{mm})$} \\
\hline Ruptured & 1.20 & 3.10 & 1.54 \\
\hline Unruptured & 1.20 & 2.50 & 1.52 \\
\hline \multicolumn{4}{|l|}{ Inflow angle $\left(^{\circ}\right)$} \\
\hline Ruptured & 93.00 & 179.40 & 143.20 \\
\hline Unruptured & 85.00 & 180.00 & 149.40 \\
\hline \multicolumn{4}{|l|}{ Dome/neck ratio } \\
\hline Ruptured & 0.68 & 4.61 & 1.97 \\
\hline Unruptured & 0.25 & 4.48 & 1.72 \\
\hline \multicolumn{4}{|l|}{ Width/neck ratio } \\
\hline Ruptured & 0.35 & 4.59 & 1.52 \\
\hline Unruptured & 0.17 & 4.73 & 1.65 \\
\hline \multicolumn{4}{|l|}{ Dome/width ratio } \\
\hline Ruptured & 0.60 & 4.88 & 1.49 \\
\hline Unruptured & 0.40 & 4.50 & 1.21 \\
\hline \multicolumn{4}{|l|}{$A_{1} / M_{1}$ ratio } \\
\hline Ruptured & 0.42 & 1.59 & 0.93 \\
\hline Unruptured & 0.48 & 1.47 & 0.89 \\
\hline \multicolumn{4}{|c|}{$\mathrm{M}_{2}$ temporal/frontal ratio } \\
\hline Ruptured & 0.63 & 1.82 & 1.03 \\
\hline Unruptured & 0.62 & 1.33 & 0.99 \\
\hline
\end{tabular}

Max $=$ maximum $; \min =$ minimum

The forest plot shows graphically the distribution of the normalized variables (Fig. 2). As can be seen in this figure, only the dome of the aneurysm is highly significant in predicting rupture. The shape of the aneurysms was 
TABLE 3. Significance analysis of variables for aneurysm rupture

\begin{tabular}{lrcc}
\hline \multicolumn{1}{c}{ Variable } & \multicolumn{1}{c}{ Mean } & SD & $p$ Value \\
\hline Dome & 0.4354726 & 0.5647044 & 0.0000069 \\
\hline Neck & 0.2420408 & 0.5397089 & 0.0047940 \\
\hline Width & 0.2455310 & 0.5595145 & 0.0056902 \\
\hline $\mathrm{ICA}$ & -0.0043371 & 0.1635126 & 0.8611639 \\
\hline $\mathrm{A}_{1}$ & 0.0389930 & 0.1840903 & 0.1671994 \\
\hline $\mathrm{M}_{1}$ & 0.0076912 & 0.1640732 & 0.7573450 \\
\hline $\mathrm{M}_{2}$ frontal & -0.0289118 & 0.2216268 & 0.3916622 \\
\hline $\mathrm{M}_{2}$ temporal & 0.0066495 & 0.1932542 & 0.8205441 \\
\hline Inflow angle & -0.0373831 & 0.3298742 & 0.4563171 \\
\hline Dome/neck ratio & 0.1914995 & 0.5257933 & 0.0200169 \\
\hline Width/neck ratio & -0.0020836 & 0.5615531 & 0.9804781 \\
\hline Dome/width ratio & 0.1911866 & 0.5437082 & 0.0244251 \\
\hline $\mathrm{A}_{1} / \mathrm{M}_{1}$ ratio & 0.0299570 & 0.2467595 & 0.4250843 \\
\hline $\mathrm{M}_{2}$ temporal/frontal ratio & 0.0356150 & 0.2853314 & 0.4122641 \\
\hline Bodfactype indicat
\end{tabular}

Boldface type indicates statistical significance.

bilaterally identical in 22 cases (50\%). In cases of asymmetrical presentation of the aneurysm shape, 19 ruptured aneurysms were irregularly shaped. This was statistically significant, as measured with the chi-square test $(\mathrm{p}=$ 0.001 ). The remaining 3 ruptured aneurysms with regular shape were, interestingly, larger than their irregular unruptured counterparts.

The size distributions of the dome, neck, and width for ruptured and unruptured aneurysms are shown in box plots (Fig. 3). For illustrative purposes, the same distributions can be presented as scatter plots and with interpolated logistic regression. It should be noted, however, that logistic regression is not a suitable tool for examining paired values, as in our case (Fig. 4).

The presented correlations indicate that the initial assumption of variable independence is probably too strong. In addition, it can be expected that the diameters of different blood vessels for a single patient are also related. This suggests that for this study, the Sidak's correction is too conservative and that the significance level can be relaxed. On the other hand, even at the commonly used level of 0.05 , only 4 variables-dome, neck, width, and the dome-to-neck ratio-would be significant. It is, however, questionable whether the significance level can be relaxed so far, because this would imply a complete redundancy between the variables. We therefore conclude that for mirror aneurysms, only the dome is an independent and reliable predictor for rupture.

\section{Discussion}

There are a number of studies focusing on anatomical and clinical risk factors for aneurysm rupture. ${ }^{6,79}$ However, there are interindividual differences like blood pressure profiles that make it difficult to establish such risk factors for each patient. Hoh et al. examined the predictive factors for rupture in 30 patients with 67 multiple aneurysms, and used these patients as internal controls. ${ }^{12}$

In our study we include only patients with mirror MCA bifurcation aneurysms, to form a homogeneous group and to enable better and easier comparison, because the aneurysms are exposed to the same physiological environment, differing only in local hemodynamic factors. Therefore, selection biases can be excluded in any pair of aneurysms. With this number of ruptured mirror aneurysms in the present study, we showed that these lesions are quite similar in their hemodynamics and differ only in some details like size and shape, which are significant predictors for the risk of rupture.

The inclination angle plays a crucial role in aneurysm rupture in some studies. ${ }^{20}$ Hence, we were surprised to find out that this factor did not play a role at all in mirror aneurysms of the MCA. The aneurysms in our study had almost the same inclination angle. The inclination angle of both mirror aneurysms was approximately $145^{\circ}$. Nikolć et al. showed that ruptured aneurysms had an inclination angle of $139^{\circ}$ compared to unruptured aneurysms, which had an inclination of $100^{\circ} .{ }^{20}$ In our study the mean inclination angle was close to the described $139^{\circ}$ for the ruptured and unruptured aneurysms.

The study of Elsharkawy et al. indicated risk factors for MCA aneurysm rupture in lesions that are 7-14 $\mathrm{mm}$, have an irregular wall, and have a height/width ratio $>1 .{ }^{5}$ Our study of mirror aneurysms is in accordance with Elsharkawy et al.'s results concerning size and irregularity, but we did not detect a difference in the height/width ratio in mirror aneurysms. The authors of the earlier study indicated that apart from the size of aneurysms, other factors (patient- and aneurysm-related) should be combined to predict rupture. When these interindividual factors are eliminated, though, as in the present study of mirror aneurysms, size and shape seem to play a role as the only predictors.

There are different theories on the cause of rupture. If we assume that the aneurysms develop at the same timeirrespective of a low or high wall shear stress - the fastergrowing one is at higher risk for rupture. Furthermore, our results are in accordance with the work of Jou and Britz, who studied a patient with 8 aneurysms and assumed that aneurysms in a single individual either have different growth rates or are at different developmental stages. Hence, the ruptured ones are either growing faster or are older. ${ }^{14}$ In their work, those authors assume that the aneurysms are influenced by the flow rate, which creates larger aneurysms. In our study the flow rates were the same, because we could find no significant differences in the anatomical features proximal and distal to the aneurysms or in the inflow angle. Nevertheless, one of the two aneurysms is larger, and eventually ruptures when it grows close to $7 \mathrm{~mm}$.

The question is what induces aneurysm growth. In the meta-analysis by Brinjikji et al., the authors identified a number of risk factors. When they excluded interindividual risk factors like female sex, smoking history, age $>50$ years, and aneurysm location, they found a higher growth rate for nonsaccular and larger aneurysms. Aneurysm growth was associated with a $3.1 \%$ rupture risk annually compared with a $0.1 \%$ risk in stable aneurysms. ${ }^{24}$ The authors also showed that $2.5 \%$ of aneurysms per year are growing, and growing aneurysms had a 30-times-higher 


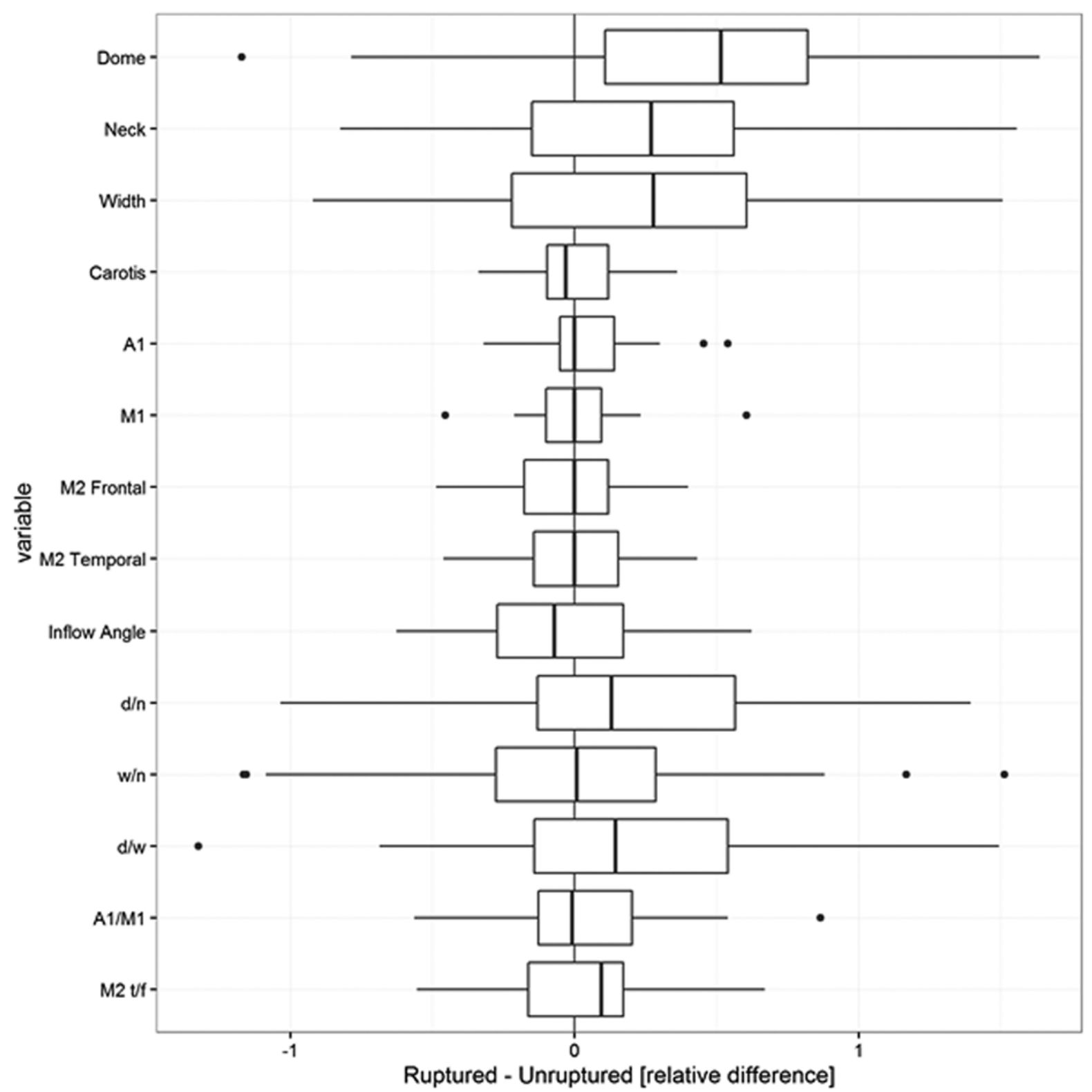

FIG. 2. Forest plot showing distribution of the normalized variables. The black circles designate outliers. Carotis $=I C A ; d / n=$ dome/neck; $\mathrm{d} / \mathrm{w}=$ dome/width; $\mathrm{t} / \mathrm{f}=$ temporal/frontal; $\mathrm{w} / \mathrm{n}=$ width/neck.

risk of rupture. This theory is confirmed by the work of Inoue et al., which followed UIAs via MRI and came to the conclusion that growth of UIAs increases the risk of rupture. Those authors indicated a rupture risk of $18.5 \%$ / person-year in growing aneurysms. ${ }^{13}$ In our study the ruptured aneurysm was 50\% larger than the unruptured one. In conclusion, it is important to understand factors that lead to aneurysm growth, because these aneurysms are less stable and tend to rupture. ${ }^{3}$ We also have a significant suspicion that the growing aneurysm is the one that ruptures first.

Wall shear stress could play a role in aneurysm formation and rupture. Jou et al. characterized wall shear stress in intracranial aneurysms in the same anatomical location but in different individuals. ${ }^{15}$ When they focused on the in- traaneurysmal flow, they showed that ruptured aneurysms had greater areas under low wall shear stress than did unruptured ones. These authors showed that the maximal wall shear stress in ruptured and unruptured aneurysms is similar and point to hemodynamic factors other than wall shear stress that are important in aneurysm rupture, although their results apply only to aneurysms on the ICA, which are under different shear stress from aneurysms to the MCA bifurcation. ${ }^{15}$

In contrast, other studies imply that a locally elevated wall shear stress contributes to focalized wall damage that forms blebs on the aneurysm wall and leads to a higher rupture risk. ${ }^{4}$ Nevertheless, irrespective of the mechanisms of development, growth, and rupture of aneurysms (which are controversial), the aneurysm wall endures on- 

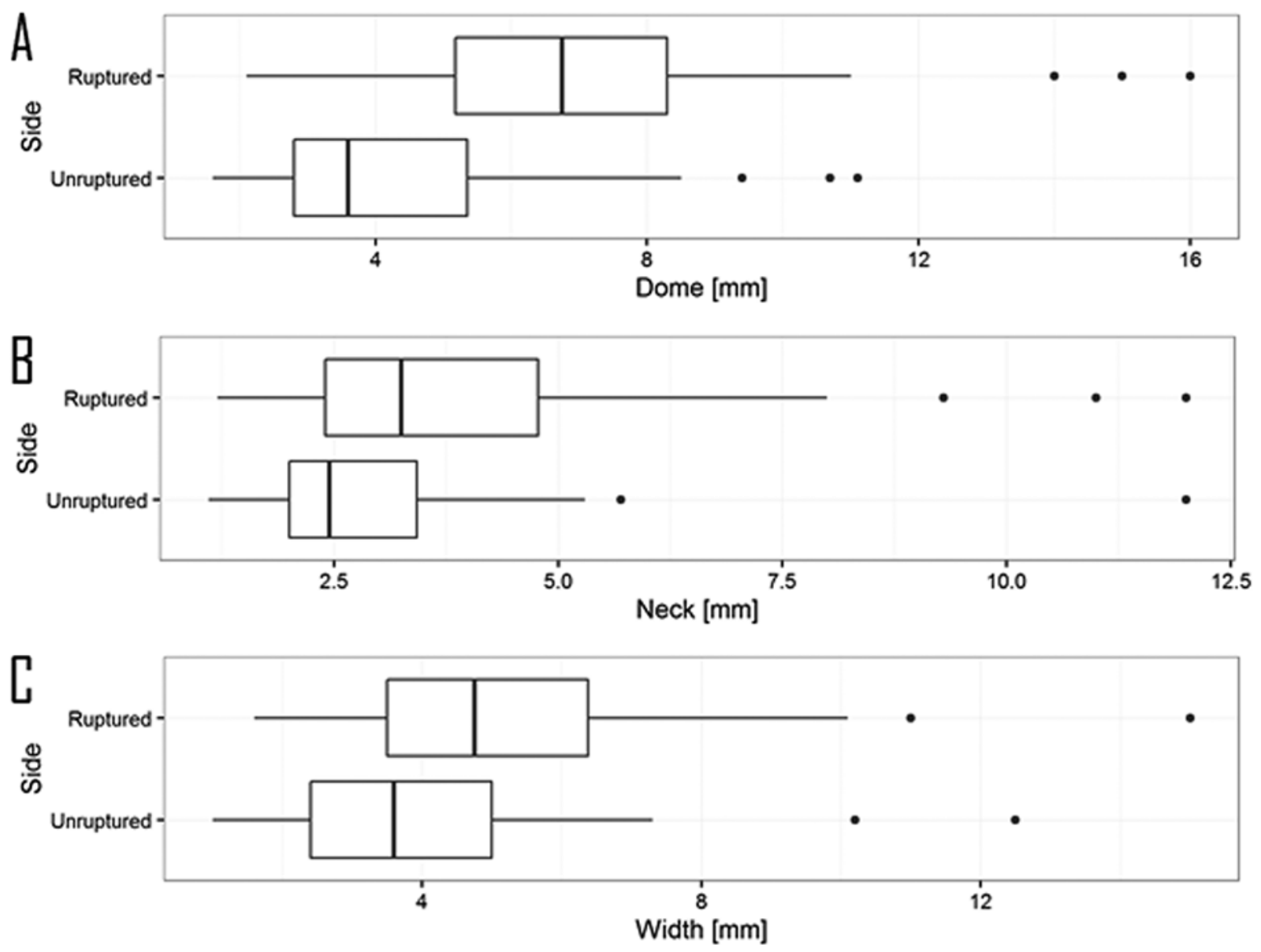

FIG. 3. Box plots showing the size distribution of the aneurysms according to the features measured. A: Dome. B: Neck. C: Width.

going injury that leads to wall failure and rupture. As in our study, a number of others show an increased risk of rupture for aneurysms containing multiple lobes.,22 $\mathrm{Ce}$ bral et al. assume that an elevated wall shear stress caused by the impact of the inflow jet causes local wall damage in the aneurysm and leads to bleb formation. ${ }^{4}$ The results of our study lead to the suspicion that (because the hemodynamics are quite similar) such a bleb forms randomly in one of the mirror aneurysms. After bleb formation or damage to the aneurysm wall, intraaneurysmal hemodynamics between the two lesions will change. The one that suffers injury of its wall first starts to grow. After this point the aneurysms are not similar anymore, and one of them becomes weak. Biomechanics and interactions with endothelial factors eventually lead to rupture. The initial wall damage could be attributed to changes in wall biology and inflammation. The creation of a bleb does not change the overall flow in the aneurysm, because the inflow angle and other factors remain the same, but the distribution of regions of abnormal wall shear stress between mirror aneurysms could change. Additionally, flow turbulences can be different between the aneurysms after bleb formation. This indicates that apart from similar hemodynamic factors, there are other factors in an aneurysm that apply stress on the aneurysm wall.
Inflammation seems to play a crucial role in this mechanism. Vakil et al. showed that contrast agent permeability across the aneurysm wall was a significant and size-independent factor for rupture risk. ${ }^{23}$ Even if the aneurysms seem to be similar in both the inclination angle and the ratios we calculated in our study, it does not mean that they are similar in histopathological features. Irregularities on their surface can be completely different because apoptosis of endothelial cells, loss of collagen, and remodeling of extracellular matrix can lead to heterogeneity of the walls, with creation of thin translucent areas that are a locus minoris resistentiae on the wall, predisposing an aneurysm to rupture.

These factors have to be taken into account as possible predictors for aneurysm rupture. We did not include them in our study because our patients were admitted to our hospital after aneurysm rupture. The intraaneurysmal flow pattern can modify the aneurysm wall in one of the mirror aneurysms without evident differences in the macrohemodynamics. Local hemodynamics in the aneurysm wall can induce vascular injury, which can cause inflammation that leads to internal elastic lamina and tunica thinning, macrophage infiltration, and extracellular matrix remodeling, which changes the wall thickness between two aneurysms that seem quite similar macroscopically and in imaging 

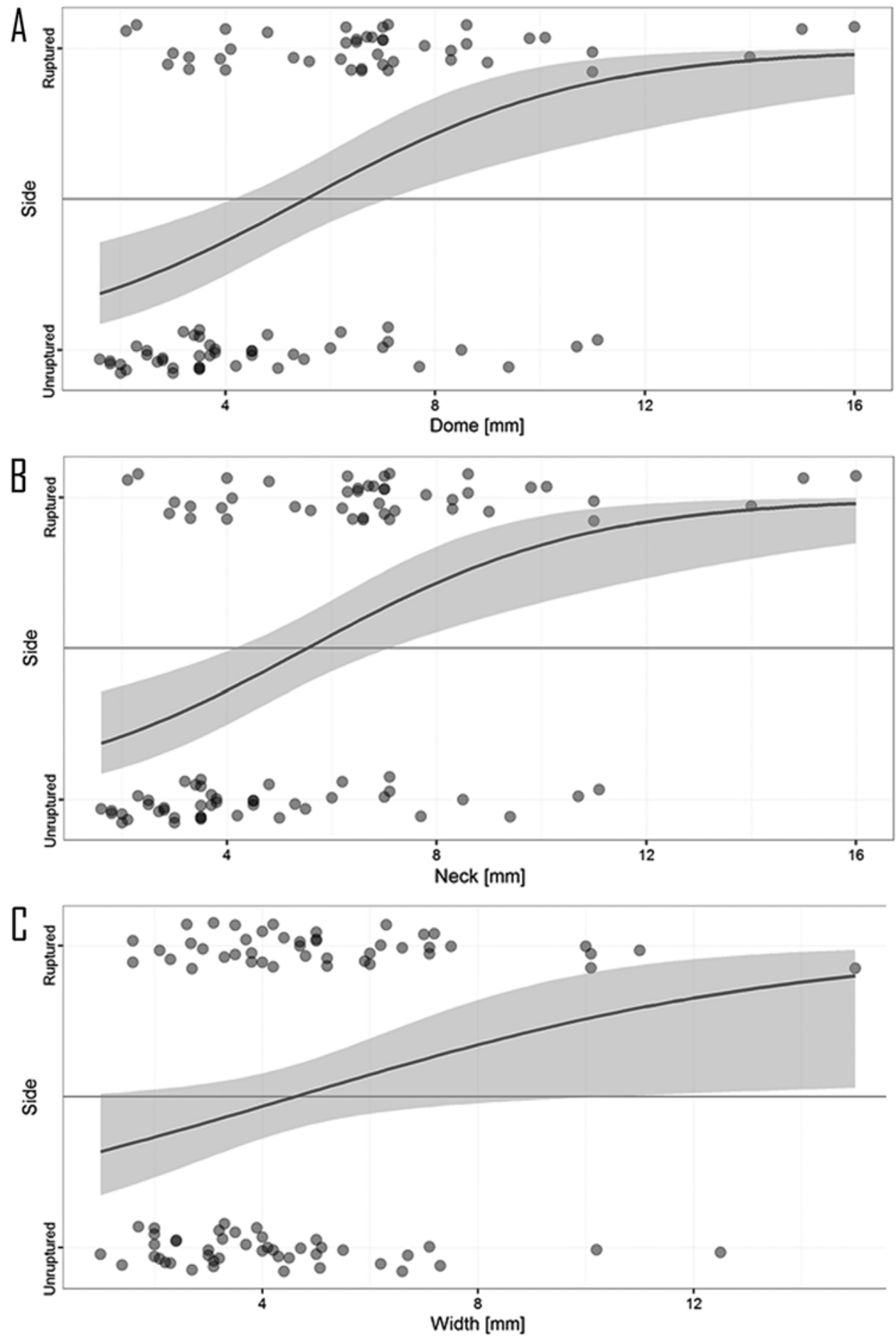

FIG. 4. Scatter plots with interpolated logistic regression showing data according to the aneurysm features measured. A: Dome. B: Neck. C: Width.

analysis. Of course these parameters are the subject of new neuroimaging techniques and cannot be specified at the moment without surgery and histological analysis, therefore falling out of the "predictors" category., ${ }^{1,10,11,17}$ In our personal experience, we had a case of a fusiform aneurysm that showed an inflamed wall on MRI and that under ther- apy with aspirin decreased in size. However this is only one case, which does not allow further assumptions.

It is difficult to predict which aneurysm will become unstable and consequently has a higher risk of rupture when mirror aneurysms are of equal size. If one of the two aneurysms starts growing, though, it can be considered 
unstable. In the case of different-sized mirror aneurysms, the larger one can be considered unstable, with a higher risk of rupture, even when we do not know its growth rate. What caused one of the two mirror aneurysms to become unstable is difficult to say, but aneurysms with a main axis parallel to the parent artery (like MCA mirror aneurysms) have a jet flow pattern and an uneven distribution of unsteady pressure, turning them into a chaotic system in which small changes can lead to instability of the aneurysm wall. ${ }^{8,21}$ Szikora et al. assumed that apart from fluid dynamics described by Laplace's law, which correlates aneurysm size with rupture risk, there should be other factors too, like intra- and extraaneurysmal flow conditions, that may play a role in the growth and rupture of aneurysms. ${ }^{21}$

Finally, we note that our study is restricted by its retrospective design. Further prospective studies on this issue might be helpful to answer the open questions. In this context it is important to mention that computational flow analysis software could be used in prospective studies to extend our pathoanatomical analysis by an additional flow analysis, which could probably bring more insights to this field.

\section{Conclusions}

In our study we show that the extraaneurysmal flow dynamics in mirror aneurysms are nonsignificant, and the aneurysmal geometry also does not seem to play a role as a predictor for rupture. The only predictors for rupture were the size and shape of the aneurysms. It seems that, under the same conditions, one of the two aneurysms suffers changes in its wall and starts growing in a more or less stochastic manner. Newer imaging methods should enable us to see which aneurysm has an unstable wall and to predict the rupture risk. At the moment we only can conclude that, in case of MCA mirror aneurysms, the larger one, with or without shape irregularities, is the unstable aneurysm and that this is the one that needs to be treated. Reaching the critical size of $7 \mathrm{~mm}$ and being approximately $50 \%$ larger than the other one should be an indication for treatment.

\section{References}

1. Aoki T, Kataoka H, Ishibashi R, Nozaki K, Egashira K, Hashimoto N: Impact of monocyte chemoattractant protein-1 deficiency on cerebral aneurysm formation. Stroke 40:942951, 2009

2. Beck J, Rohde S, el Beltagy M, Zimmermann M, Berkefeld $\mathrm{J}$, Seifert V, et al: Difference in configuration of ruptured and unruptured intracranial aneurysms determined by biplanar digital subtraction angiography. Acta Neurochir (Wien) 145:861-865, 2003

3. Brinjikji W, Zhu YQ, Lanzino G, Cloft HJ, Murad MH, Wang $Z$, et al: Risk factors for growth of intracranial aneurysms: a systematic review and meta-analysis. AJNR Am J Neuroradiol 37:615-620, 2016

4. Cebral JR, Sheridan M, Putman CM: Hemodynamics and bleb formation in intracranial aneurysms. AJNR Am J Neuroradiol 31:304-310, 2010

5. Elsharkawy A, Lehečka M, Niemelä M, Kivelev J, BillonGrand R, Lehto H, et al: Anatomic risk factors for middle cerebral artery aneurysm rupture: computed tomography angiography study of 1009 consecutive patients. Neurosurgery 73:825-837, 2013
6. Etminan N, Beseoglu K, Barrow DL, Bederson J, Brown RD $\mathrm{Jr}$, Connolly ES Jr, et al: Multidisciplinary consensus on assessment of unruptured intracranial aneurysms: proposal of an international research group. Stroke 45:1523-1530, 2014

7. Fan J, Wang Y, Liu J, Jing L, Wang C, Li C, et al: Morphological-hemodynamic characteristics of intracranial bifurcation mirror aneurysms. World Neurosurg 84:114-120, 120. e1-120.e2, 2015

8. Foutrakis GN, Yonas H, Sclabassi RJ: Saccular aneurysm formation in curved and bifurcating arteries. AJNR Am J Neuroradiol 20:1309-1317, 1999

9. Greving JP, Wermer MJH, Brown RD Jr, Morita A, Juvela $\mathrm{S}$, Yonekura M, et al: Development of the PHASES score for prediction of risk of rupture of intracranial aneurysms: a pooled analysis of six prospective cohort studies. Lancet Neurol 13:59-66, 2014

10. Hasan DM, Mahaney KB, Magnotta VA, Kung DK, Lawton MT, Hashimoto T, et al: Macrophage imaging within human cerebral aneurysms wall using ferumoxytol-enhanced MRI: a pilot study. Arterioscler Thromb Vasc Biol 32:1032-1038, 2012

11. Hashimoto T, Meng H, Young WL: Intracranial aneurysms: links among inflammation, hemodynamics and vascular remodeling. Neurol Res 28:372-380, 2006

12. Hoh BL, Sistrom CL, Firment CS, Fautheree GL, Velat GJ, Whiting JH, et al: Bottleneck factor and height-width ratio: association with ruptured aneurysms in patients with multiple cerebral aneurysms. Neurosurgery 61:716-723, 2007

13. Inoue T, Shimizu H, Fujimura M, Saito A, Tominaga T: Annual rupture risk of growing unruptured cerebral aneurysms detected by magnetic resonance angiography. J Neurosurg 117:20-25, 2012

14. Jou L, Britz G: Correlation between aneurysm size and hemodynamics in one individual with multiple small intracranial aneurysms. Cureus 8:e683, 2016

15. Jou LD, Lee DH, Morsi H, Mawad ME: Wall shear stress on ruptured and unruptured intracranial aneurysms at the internal carotid artery. AJNR Am J Neuroradiol 29:1761-1767, 2008

16. Macdonald RL, Schweizer TA: Spontaneous subarachnoid haemorrhage. Lancet 389:655-666, 2017

17. Mantha A, Karmonik C, Benndorf G, Strother C, Metcalfe $\mathrm{R}$ : Hemodynamics in a cerebral artery before and after the formation of an aneurysm. AJNR Am J Neuroradiol 27:1113-1118, 2006

18. Maslehaty H, Ngando H, Meila D, Brassel F, Scholz M, Petridis AK: Estimated low risk of rupture of small-sized unruptured intracranial aneurysms (UIAs) in relation to intracranial aneurysms in patients with subarachnoid haemorrhage. Acta Neurochir (Wien) 155:1095-1100, 2013

19. Morita A, Kirino T, Hashi K, Aoki N, Fukuhara S, Hashimoto N, et al: The natural course of unruptured cerebral aneurysms in a Japanese cohort. N Engl J Med 366:2474-2482, 2012

20. Nikolć I, Tasić G, Antunović V, Rakić M, Mihajlović M, Joković M, et al: Comparative analysis of the animal model and results of the clinical research of the aneurysm inclination angle as the predisposing factor for the occurrence of rupture. Srp Arh Celok Lek 141:150-154, 2013

21. Szikora I, Paal G, Ugron A, Nasztanovics F, Marosfoi M, Berentei Z, et al: Impact of aneurysmal geometry on intraaneurysmal flow: a computerized flow simulation study. Neuroradiology 50:411-421, 2008

22. Tsukahara T, Murakami N, Sakurai Y, Yonekura M, Takahashi $\mathrm{T}$, Inoue $\mathrm{T}$, et al: Treatment of unruptured cerebral aneurysms; a multi-center study at Japanese national hospitals. Acta Neurochir Suppl 94:77-85, 2005

23. Vakil P, Ansari SA, Cantrell CG, Eddleman CS, Dehkordi $\mathrm{FH}$, Vranic J, et al: Quantifying intracranial aneurysm wall 
permeability for risk assessment using dynamic contrastenhanced MRI: a pilot study. AJNR Am J Neuroradiol 36:953-959, 2015

24. Wiebers D, Whisnant J, Forbes G, Meissner I, Brown R Jr, Piepgras D, et al: Unruptured intracranial aneurysms-risk of rupture and risks of surgical intervention. N Engl J Med 339:1725-1733, 1998

25. Wiebers DO, Whisnant JP, Huston J III, Meissner I, Brown RD Jr, Piepgras DG, et al: Unruptured intracranial aneurysms: natural history, clinical outcome, and risks of surgical and endovascular treatment. Lancet 362:103-110, 2003

\section{Disclosures}

The authors report no conflict of interest concerning the materials or methods used in this study or the findings specified in this paper.

\section{Author Contributions}

Conception and design: Maslehaty, Petridis. Acquisition of data:
Maslehaty, Capone, Frantsev, Jabbarli, Petridis. Analysis and interpretation of data: Maslehaty, Capone, Frantsev, Petridis. Drafting the article: Maslehaty, Petridis. Critically revising the article: Jabbarli, Cornelius, Kamp, Cappabianca, Sure, Steiger, Petridis. Reviewed submitted version of manuscript: all authors. Approved the final version of the manuscript on behalf of all authors: Maslehaty. Statistical analysis: Fischer. Administrative/ technical/material support: Fischer. Study supervision: Maslehaty, Petridis.

\section{Supplemental Information}

\section{Online-Only Content}

Supplemental material is available with the online version of the article.

Supplemental Table 1. https://thejns.org/doi/suppl/10.3171/ 2017.2.JNS162705

\section{Correspondence}

Homajoun Maslehaty, Department of Neurosurgery, University Hospital Essen, Hufelandstrasse 55, Essen 45122, Germany. email: h.maslehaty@gmx.de. 